\title{
The plasticity of the perceptual process
}

\author{
FELIX E. GOODSON \\ DePauw University, Greencastle, Indiana 46135
}

\begin{abstract}
After only $2 \mathrm{~h}$ of tracing star patterns while viewing their progress in a mirror, many subjects demonstrated an automatic and irresistible inversion in handwriting. These findings suggest that the process underlying perceptual organization is extremely plastic and virtually rules out the possibility of demonstrating that such phenomena as size constancy, shape constancy, perceived depth, and so on, are due solely to innate factors.
\end{abstract}

For many years at DePauw University, students in the introductory psychology course participated in a mirror drawing experiment. Each student spent $2 \mathrm{~h}$ tracing star patterns that were hidden from direct observation and visible to them only in a mirror. Since input from the visual and tactual modalities were in opposition to that which was normal, the going was very difficult in the beginning, but as the trials progressed, most students became able to trace the star patterns rapidly and with few errors.

Mirror drawing experiments, of course, are not unusual in themselves, but certain effects discovered and replicated year after year are not only remarkable but provide important information about the process that underlies perceptual organization. After only $2 \mathrm{~h}$ of tracing star patterns while looking in a mirror, between $10 \%$ and $20 \%$ of all students experienced a mirror image reversal in writing. In spite of their efforts to keep from doing so, they wrote their signatures, for instance, in mirror image. In a few cases, the influence was so pronounced that the students wrote in mirror images for a number of pages.

Fortunately, the effect does not last long. Without exception, all students were back to normal within a few hours. But the occurrence of hundreds of such cases suggests that: (1) the process underlying perceptual organization is extremely plastic, in that it can be profoundly influenced within a very short time frame, (2) the process appears to be highly generalizable (i.e., once the cues from eye and hand have become restructured in the mirror drawing situation, this new structure can be transferred to writing), (3) the process appears to involve a central restructuring rather than dependence on specific cues (i.e., the movements involved in writing, and, presumably, the kinesthetic inputs, are much more

Reprint requests should be sent to the author, Department of Psychology, DePauw University, Greencastle, Indiana 46135. complex, as well as being different from those involved in tracing a star pattern), (4) the process apparently is unconscious and nonvolitional (i.e., the students were unaware of the perceptual reorganization that was taking place, and once it had taken place, the restructuring was automatically imposed, in spite of the students' efforts to keep it from happening), (5) the aspect of the process having to do with visual structuring appears to be more stable than that having to do with tactual structuring (i.e., although tactual inversion was common, visual inversion was never demonstrated). This conclusion is highly debatable, since the experimental situation provided many more occasions for reaffirming the original visual organization than were available for original tactual organization (i.e., the subject's hand was almost completely restricted to the drawing behavior, while his eyes were often free to glance about the room).

The fact that a generalized inversion in perceptual organization can be produced by only $2 \mathrm{~h}$ of mirror drawing has important implications for research. It suggests that it will be difficult, if not impossible, to demonstrate that any type of perceptual phenomena, whether size constancy or shape constancy or the perception of depth, is contributed solely by innate factors. The process underlying perceptual organization is apparently so plastic that even a short period of appropriate experience is sufficient to produce critical changes. Thus, by the time an infant is even a few hours or days old, the experience necessary for perceptual organization could already have occurred. In short, if an infant has matured enough to make the responses necessary for demonstrating a perceptual phenomenon, he has very likely already had more than sufficient time for such organization to have been derived from experience.

(Received for publication January 13, 1981.) 